\title{
$\mathrm{WiBro}$ 에서 마코프 체인을 이용한 성능분석
}

\author{
박원길 ${ }^{1}$, 김형진 $2^{*}$ \\ ${ }^{1}$ 성균관대학교 유비쿼터스 컴퓨팅 기술연구소, ${ }^{2}$ 전북대학교 응용시스템공학부
}

\section{Performance Analysis using Markov chain in WiBro}

\author{
Won-Gil Park ${ }^{1}$ and Hyoung-Jin Kim ${ }^{2 *}$ \\ ${ }^{1}$ Ubiquitous Computing Technology Research Institute, Sunkyunkwan University \\ ${ }^{2}$ Division of Applied System Engineering, Chonbuk National University
}

\begin{abstract}
요 약 WiBro의 ACR(Access Control Router)은 ACR간을 움직일 때만 홈 에이전트와 상대 노드에게 위치등록을 하 므로, 위치등록비용은 MIPv6에 비해 적지만, 이동 노드의 송수신되는 모든 패킷들은 $\mathrm{ACR}$ 을 통해 전달되기 때문에 $\mathrm{ACR}$ 이 관리하는 이동노드의 수가 많아지면 $\mathrm{ACR}$ 의 패킷처리비용은 증가한다. 따라서 $\mathrm{ACR}$ 도메인 내에 있는 이동 노 드들을 관리하는 $\mathrm{ACR}$ 이 잘 작동한다면 $\mathrm{ACR}$ 도메인 내의 통신상태는 원활하다. 그러나 $\mathrm{ACR}$ 이 잘 작동하지 않으면 네트워크의 delay가 발생하므로 $\mathrm{ACR}$ 의 역할은 중요하다. 본 논문에서는 $\mathrm{WiBro}$ 의 효율적인 작동을 위해 $\mathrm{ACR}$ 의 성능 을 측정한다. 이를 위해, 성능평가 요소로서 이동노드의 거부확률(deny probability)와 ACR 성능의 총이익을 사용한다. 그리고 이동모델로서 랜덤워크 모델을 이용한다.
\end{abstract}

\begin{abstract}
The ACR (Access Control Router) of WiBro processes location registration of the Correspondent Node and Home Agent as the Correspondent Node moves between ACRs. Therefore, the location update cost is low compared with MIPv6. However, all packets which are sent and received are sent through the ACR, so as the number of mobile nodes that are managed by the ACR increases, the cost of packet delivery also increases. Therefore, the communication state of the ACR domain remains smooth when the ACR which manages the mobile node in the ACR domain has good performance. However, network delays occur unless the ACR performs well, so the role of the ACR is important. In this paper, we analysis performance of the ACR for efficient realization of the WiBro standard. By using the Deny Probability and the Total Profit of ACR performance and apply it to the Random Walk Mobility model as the mobility model.
\end{abstract}

Key Words : WiBro, Access Control Router, Deny probability, Total profit

\section{1. 서론}

IP기반 무선망에서 실시간 서비스를 지원하기 위한 이 동성 관리에 대한 중요성이 커짐에 따라 Internet Engineering Task Force(IETF)에서는 IPv6이동성 관리 프 로토콜인 Mobile IPv6(MIPv6)를 제안하였다[1]. 그러나 이 방식은 이동 단말이 다른 IP 프리픽스 도메인(Prefix Domain)으로 이동할 때마다 새로운 주소( $\mathrm{NCoA})$ 를 자신 의 홈 에이전트(HA:Home Agent) 및 상대노드 (CN:Correspond Node)와 갱신하는 과정을 수행해야한다. 이러한 갱신과정은 이동 노드가 빈번하게 이동할수록 이
동 노드의 위치등록을 위한 오버헤드가 증가한다. 이러한 문제점을 해결하기 위해 나온 것이 HMIPv6(Hierarchical MIPv6) 와 FMIPv6(Fast Handover for MIPv6)이다.

HMIPv6은 MAP(Mobility Anchor Point)라는 에이전트 를 추가하여 지역이동성을 문제를 해결하였다[2]. FMIPv6은 링크계층(Layer 2)의 트리거(Trigger0기법을 이용한 예측된 빠른 핸드오버 처리와 패킷 손실 방지를 위한 터널기반의 핸드오버를 지원한다[3].

IPv6 기반인 WiBro(Wireless Broadband)는 IEEE 802.16 표준에서 $\mathrm{PHY}$ (physical)에 $\mathrm{OFDM}$ (Orthogonal Frequency Division Multiplexing)이 적용된 2.3ㅂtz의 대역

*교신저자 : 김형진(kim@chonbuk.ac.kr)

접수일 09년 11월 02일 수정일 10년 01월 05일 게재확정일 10년 01월 20일 
을 사용하며, PSS(Portable Subscriber Station)가 정지 및 이동 중에서도 언제, 어디서나 중속으로 무선인터넷 접속 이 가능한 서비스이다. WiBro 서비스의 특징은 정지 및 보행뿐만 아니라 중속의 이동성 및 상향링크 $1 \mathrm{Mbps}$, 하 향링크 $3 \mathrm{Mbps}$ 의 데이터 전송률을 지원한다 $[4,5]$. 모바일 이 움직일때마다 홈에이전트와 상대노드에게 위치등록을 하는 MIPv6에 비해 WiBro는 ACR 간을 이동할때만 위 치 등록을 하기 때문에 위치등록비용이 작다. 그러나 이 동노드로 송수신되는 모든 패킷들은 ACR을 통해야 하기 때문에 $\mathrm{ACR}$ 이 관리하는 이동노드의 수가 많아지면 $\mathrm{ACR}$ 의 패킷 처리비용이 증가한다. ACR은 ACR 도메인 내에 있는 이동 노드들을 관리한다. 그러므로 $\mathrm{ACR}$ 이 잘 작동 한다면 ACR 도메인 내의 통신상태는 원하지만 ACR이 잘 작동하지 않으면 네트워크의 지연이 발생하게 되므로 $\mathrm{ACR}$ 의 역할은 중요하다. 본 논문에서는 WiBro 의 효율 적인 작동을 위해서 $\mathrm{ACR}$ 의 성능을 측정한다.

성능평가 요소로서 이동노드의 거부 확률과 $\mathrm{ACR}$ 성능 을 이용한 MIPv6 총비용과 WiBro의 총비용의 차인 총이 익을 사용하고, 이동모델은 랜덤워크 모델을 이용한다.

본 논문의 구성은 다음과 같다. 2장에서는 관련연구인 MIP6와 WiBro 의 기술에 대해 알아보고, 이동모델인 랜 덤워크 모델을 서술한다. 3장에서는 마코프 체인을 사용 하여 $\mathrm{ACR}$ 의 성능분석을 위한 분석 모델을 정의한다. 4 장에서는 성능분석에 대한 결과를 서술하고, 5 장에서 결 론을 맺는다.

\section{2. 관련연구}

\subsection{MIPv6}

$\mathrm{MIPv6}$ 는 이동 노드의 이동에 상관없이 항상 인터넷 서비스를 제공하기위한 프로토콜이다[1].

$\mathrm{AR}$ (Access Router)는 이동노드에게 서비스를 제공하 기 위한 무선중계기로, 이동노드가 새로운 $\mathrm{AR}$ 로 이동할 때마다 $\mathrm{CoA}$ (Care-of Address)를 다시 구성한다. 이 $\mathrm{CoA}$ 는 홈 네트워크가 아닌 다른 네트워크에서 인터넷 서비 스를 제공받기 위해 사용되는 이동 노드의 임시주소이다. 이때 이동 노드는 $\mathrm{AR}$ 주소의 Prefix를 이용하여 $\mathrm{CoA}$ 를 구성한다. 이동노드가 다른 $\mathrm{AR}$ 로 이동하여 새로 생성한 $\mathrm{CoA}$ 를 홈 에이전트와 현재 통신하고 있는 상대노드에게 알린다.

그림 1은 MIPv6의 기본동작과정을 나타낸다. 새로운 $\mathrm{AR}$ 로 이동한 이동 노드는 위치등록 메시지인 $\mathrm{BU}$ (Binding Update)를 홈 에이전트에게 보낸다. 그러면
홈 에이전트는 $\mathrm{BU}$ 에 대한 응답으로 $\mathrm{BA}$ (Binding Acknowledgement)를 이동노드에게 보낸다. 마찬가지로 상대노드에게 $\mathrm{BU}$ 을 보내고 $\mathrm{BA}$ 을 받으므로 이동노드는 상대노드와 위치등록을 한다. MIPv6은 이동성을 가진 모 바일 노드가 새로운 AR로 이동할 때마다 이동노드의 위 치를 홈 에이전트와 상대노드에게 항상 위치를 등록해야 한다는 문제점을 가지고 있다.

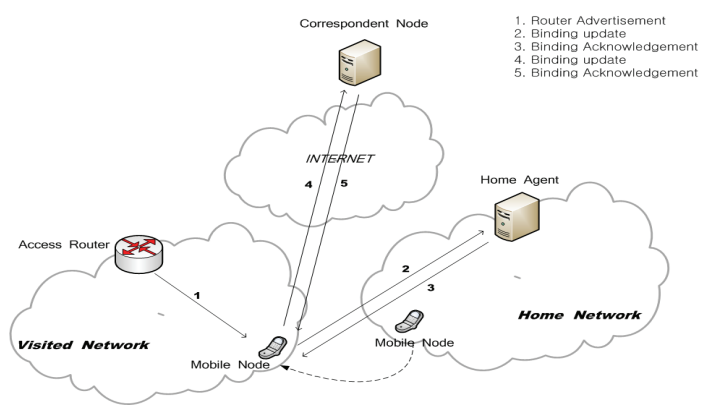

[그림 1] MIPv6 구조

\subsection{WiBro (Wireless Broadband)}

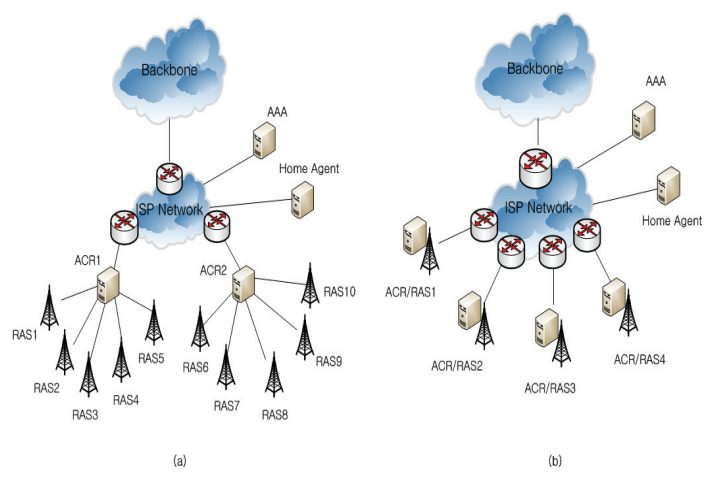

[그림 2] WiBro 구조

PSS(Portable Subscriber Staton)가 정지 및 이동 중에 도 언제, 어디서나 중속으로 무선 인터넷 접속이 가능한 서비스로 노트북 및 $\mathrm{PDA}$ 의 사용이 보편화 되면서 관심 을 받는 기술로IEEE 802.16 표준에서 PHY(Physical)에 OFDM(Orthogonal Frequency Division Multiplexing)이 적용된 $2.3 \mathrm{GHz}$ 의 대역을 사용한다. WiBro의 특징은 정 지 및 보행중에 중속의 이동성 및 상향링크 $1 \mathrm{Mbps}$, 하향 링크 $3 \mathrm{Mbps}$ 의 데이터 전송률을 지원한다.

WiBro는 다음과 같은 PSS, RAS, ACR의 구성요소를 가진다. PSS(Portable Subscriber Station)은 가입자가 휴 대 인터넷 서비스를 제공받기 위해 사용하는 기기이다. RAS(Radio Access Station)은 유선네트워크 종단에서 무 
선 인터페이스를 통하여 PSS와 송수신을 하는 구성요소 이다. ACR(Access Control Router)sms PSS와 RAS를 제 어하고 IP패킷을 라우팅하는 구성요소이다. 그림2는 WiBro 구조를 보여준다.

\section{3 이동모델}

본 논문에서는 이동모델로 랜덤워크 모델을 사용한다 [6-10].각각의 ACR 도메인의 크기가 같고 ACR 도메인은 그림 3과 같이 Hexagonal cellular 네트워크 구조로 되어 있다고 가정한다. 가장 안쪽에 있는 셀을 ' 0 '이라고 하고, ' 0 ' 셀 주위의 셀을 ' 1 '이라고 부르고, 비슷하게 2, 3으로 번호를 매긴다. 따라서 $\mathrm{r}$ 번째 링의 셀의 개수는 $6 \mathrm{r}$ 이기 때 문에 셀의 개수 $\mathrm{N}(\mathrm{D})$ 은 식 (1)과 같다.

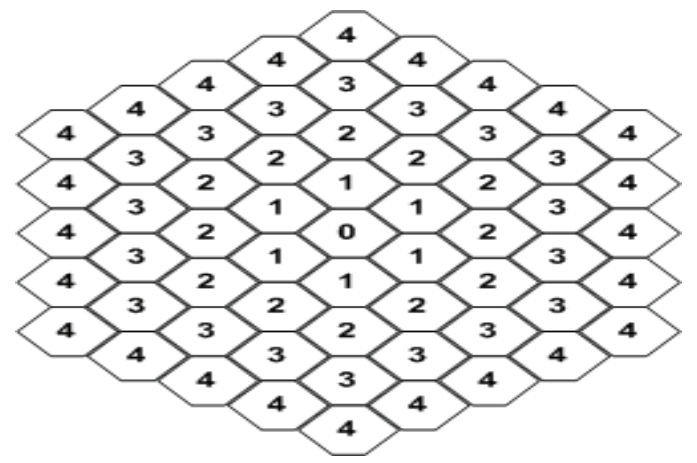

[그림 3] Hexagonal cellular 네트워크 구조

$$
N(D)=1+6 \cdot \sum_{d=1}^{D} d=1+3 \cdot D \cdot(D+1)
$$

그림3에서 이동노드가 $\mathrm{d}$ 번 셀에 위치하여 다른 셀로 이동할 경우, 중앙 셀로부터 거리가 늘어나거나 줄어들 확률은 다음과 같다.

$$
P^{+}(d)=\frac{2 d+1}{6 d} \quad \text { and } \quad P^{-}(d)=\frac{2 d-1}{6 d}
$$

본 논문에서 마코프 체인의 상태 $\mathrm{d}(\mathrm{d}>=0)$ 은 이동 노드 가 현재 위치한 셀과 중앙 셀 간의 거리로 정의한다. 따 라서 상태 $\mathrm{d}$ 은 이동 노드가 위치한 링의 색인과 같고 이 동 노드가 $\mathrm{d}$ 번째 링에 있다면 이동 노드는 상태 $\mathrm{d}$ 에 있다 고 말할 수 있다. 상태전이확률 $\alpha_{d},_{d+1}$ 와 $\beta_{d, d-1}$ 은 이동 노드가 현재 위치한 셀과 ACR 도메인의 중앙 셀 간이 거 리가 각각 증가하고 감소하는 확률이라고 정의한다. 그리
고 q는 이동 노드가 현재 셀에서 움직이지 않고 머무는 확률이라 정의한다. 그러면 식(2)에 의해서 각각의 상태 전이확률은 다음과 같이 유도 된다.

$$
\begin{aligned}
& \alpha_{d, d+1}=\left\{\begin{array}{ccc}
(1-q) & \text { if } & d=0 \\
(1-q) p^{+}(d) & \text { if } & 1 \leq d \leq D
\end{array}\right. \\
& \beta_{d, d-1=(1-q) p^{-}(d) \quad \text { if } \quad 1 \leq d \leq D}
\end{aligned}
$$

식(3)과 식(4)에 의해 ACR도메인 내에서 상태 d에 대 한 정상상태 확률(steady-state probability)는 식(5)와 같 다.

$$
P_{d, D}=P_{0, d} \prod_{i=0}^{d-1} \frac{\alpha_{i, i+1}}{\beta_{i+1, i}} \text { for } 1 \leq d \leq D
$$

식 $\sum_{d=0}^{D} P_{d, D}=1$ 이므로 이동 노드가 상태 0 에 있을 확 률은 식 (6)과 같다.

$$
P_{0, D}=\frac{1}{1+\sum_{d=1}^{D} \prod_{i=0}^{d-1} \frac{\alpha_{i, i+1}}{\beta_{i+1, i}}}
$$

\section{3. 제안기법}

홈 에이전트는 자신의 네트워크 안에 있는 어떠한 외 부 노드에게도 인터넷 연결성을 제공하지 않는다. 이는 홈 에이전트가 관리하는 이동노드의 수가 고정되어 있기 때문이다. 이에 반해 WiBro에서 ACR(Access Control Router)은 ACR 도메인 안에 있는 모든 노드들에게 인터 넷 연결성을 제공한다. 이 말은 $\mathrm{ACR}$ 이 관리하는 이동 노 드의 개수가 정해져 있지 않다는 것이다. 따라서 ACR 도 메인안에서 이동 노드의 개수가 증가하여 $\mathrm{ACR}$ 이 더 이 상의 이동 노드에게 인터넷 연결성을 제공해 줄 수 없으 면, ACR 도메인 안으로 새로 들어오는 이동노드는 $\mathrm{ACR}$ 에게 연결성을 제공받을 수 없다.

본 논문에서는 이러한 경우가 발생할 확률을 거부 확 률(deny probability)라고 한다. 거부 확률을 줄이기 위해 서는 $\mathrm{ACR}$ 의 성능을 올림으로서 해결할 수 있지만, 이는 리소스의 낭비가 발생한다. 따라서 본 논문에서는 $\mathrm{ACR}$ 도메인 안으로 들어오는 이동노드의 도착율과 거부확률 
을 고려하여 $\mathrm{ACR}$ 의 최적 성능을 구한다.

\section{1 가정(Assumption)}

분석모델을 위한 마코프 체인 모델을 세우기 위해 다 음과 같은 가정을 한다

(1) 이동노드의 도착과정은 평균 $\lambda$ 의 포아송 분포를 따른다

(2) 이동노드를 위한 서비스 시간은 평균 $\mu$ 의 지수분 포를 따른다

(3) $\mathrm{ACR}$ 의 용량은 $\mathrm{N}$ 으로 나타내며, $\mathrm{ACR}$ 이 제공할 수 있는 이동노드의 최대 개수를 나타낸다

분석모델을 위한 정의와 기호를 다음과 같이 정의한 다.

MIPv6의 총비용은 이동 노드가 ACR 도메인에 머무 는 동안 MIPv6로 인터넷 서비스를 제공받기 위해 사용되 는 비용으로, $C_{M I P}$ 로 표시하고, WiBro의 총비용은 이동 노드가 WiBro로 인터넷 서비스를 제공받기 위해 사용되 는 비용으로, $C_{W i B r o}$ 로 표기한다. 그리고 Wibro를 사용 함으로서 하나의 ACR 도메인 내에서 네트워크가 얻는 이익은 $C_{M I P}-C_{W i B r o}$ 이고, 본 논문에서는 이것을 총 이 익이라 한다.

\section{2 마코프 체인(Markov chain)분석}

그림 4 는 WiBro의 마코프 체인에서 상태전이 다이어 그램을 나태낸것이다. 그림 4 에서 $\lambda$ 는 $\mathrm{ACR}$ 도메인 안 으로 들어오는 이동 노드의 도착율을 의미하고, $\mu$ 는 이 동노드가 ACR 도메인에서 밖으로 나가는 출발율을 의미 한다. 따라서 $\mu$ 는 $\mathrm{ACR}$ 의 성능을 나타낸다.

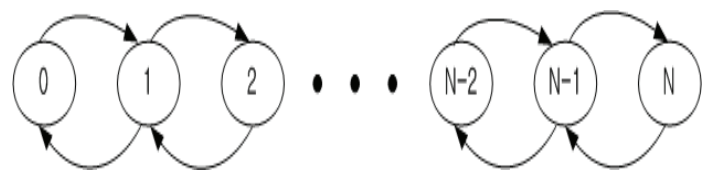

[그림 4] WiBro 마코프 체인 모델

상태 $i$ 는 $\mathrm{ACR}$ 도메인 내에 있는 이동 노드의 개수를 나타내고, $q(i, j)$ 는 상태 $i$ 에서 상태 $j$ 로 이동하는 전 이율을 의미한다. 마코프 체인의 상태 전이율은 다음과 같다.

$$
q(i, i+1)=\lambda \quad(0 \leq i\langle N)
$$

$$
q(i+1, i)=\mu \quad(0 \leq i\langle N)
$$

$q(i, i+1)$ 은 상태 $i$ 에서 상태 $i+1$ 로 가는 전이율이 고, $q(i+1, i)$ 는 상태 $i+1$ 에서 상태 $i$ 로 가는 전이율이 다. 마코프 체인의 rate-equality principle은 다음과 같은 식을 산출한다.

$$
\begin{aligned}
& \lambda P_{0}=\mu P_{1} \\
& (\lambda+\mu) P_{n}=\lambda P_{n-1}+\mu P_{n+1}(1 \leq n \leq N-1) \\
& \mu P_{N}-\lambda P_{N-1}
\end{aligned}
$$

식(8)와 $\sum_{n=0}^{N} P_{n}=1$ 에 의해 정상상태확률 $\left(P_{k}\right)$ 는 다음 과 같이 유도된다.

$$
\begin{aligned}
& P_{i}=\left(\frac{\lambda}{\mu}\right)^{i} P_{0} \\
& P_{O}= \begin{cases}\frac{1}{N} & , \lambda=\mu \\
\left(\sum_{k=0}^{N}\left(\frac{\lambda}{\mu}\right)^{k}\right)^{-1} & , \lambda \neq \mu\end{cases}
\end{aligned}
$$

$P_{N}$ 은 $\mathrm{ACR}$ 도메인 안에 이동 노드들의 수가 꽉 차 있 는 상태의 확률이므로 실질적으로 $\mathrm{ACR}$ 도메인 안으로 들어오는 이동 노드의 도착율은 $\lambda\left(1-P_{N}\right)$ 이다.

각각의 이동 노드는 $\mathrm{ACR}$ 도메인 안에서 $C_{M I P}$ 만큼 비 용을 지불하기 때문에 단위 시간당 네트워크가 얻는 비 용은 $C_{M I P} \cdot \lambda\left(1-P_{N}\right)$ 이다. 그리고 이동 노드에게 인터 넷 연결성을 제공하기 위해 사용되는 비용은 $C_{W i B r o}$ 이기 때문에 네트워크가 지불하는 비용은 $C_{W i B r o} \cdot \mu$ 이다. 따 라서 단위시간당 $\mathrm{ACR}$ 도메인 내의 총 이익 $\left(T_{P}\right)$ 은 다음 과 같다.

$$
T_{p}(\lambda, \mu)=\lambda\left(1-p_{N}\right) \cdot C_{M I P}-\mu \cdot C_{W i B r o}
$$

이동 노드의 거부 확률 $(B(\lambda, \mu))$ 은 다음과 같다. 


$$
B(\lambda, \mu)=\left(\frac{\lambda}{\mu}\right)^{N} \cdot P_{0}
$$

$\mathrm{ACR}$ 의 개수를 하나로 가정하였을 때, $\mathrm{ACR}$ 의 사용률 은 식 (12)과 같다.

$$
\rho_{M A P}=\frac{\lambda\left(1-P_{N}\right)}{\mu}
$$

이동 노드가 다른 ACR 도메인 안으로 들어갔을 때, 이동 노드는 홈에이전트와 상대노드에게 자신의 위치를 등록한다. 다음에 서술하는 랜덤워크 모델에서 $P_{D, D}$ 은 이동 노드가 상태 $\mathrm{D}$ 에 있을 정상상태 확률이므로 랜덤워 크 모델을 사용했을 경우, 이동 노드가 다른 ACR도메인 으로 이동하여 홈 에이전트와 상대 노드에게 자신의 위 치를 등록할 확률은 $P_{D, D} \cdot \alpha_{D, D+1}$ 이다. 따라서 $\mathrm{T}$ 는 이 동 노드가 각 셀에 머무는 평균시간이라 가정하면, 랜덤 워크 모델에서 단위시간 당 $\mathrm{ACR}$ 도메인 안으로 들어오 는 이동 노드의 도착율은 다음과 같다.

$$
\lambda=\frac{P_{D, D} \cdot \alpha_{D, D+1}}{T}
$$

\subsection{MIPv6와 WiBro의 총비용}

무선/모바일 네트워크의 성능을 분석하기 위하여, location update 와 packet delivery cost로 구성된 total coast가 고려되어 진다. 제안된 방법에서 MIPv6와 $\mathrm{WiBro}$ 각각에서 location update cost 와 packet delivery cost는 각각 CMlocation, CWlocation, CMpacket, CWWpacket 으로 각각 나타낸다. MIPv6와 WiBro 의 total $\operatorname{cost}(\mathrm{CMtotal}, \mathrm{CWtotal})$ 은 다음과 같이 획득되어 진다.

$$
\begin{aligned}
& C_{\text {Mtotal }}=C_{\text {Mlocation }}+C_{\text {Mpacket }} \\
& C_{\text {Wtotal }}=C_{\text {Wocation }}+C_{\text {WWpacket }}
\end{aligned}
$$

\subsection{1 location update cost}

$\mathrm{MN}$ 가 새로운 $\mathrm{AR}$ 로 이동했을 때, $\mathrm{MN}$ 은 새로운 $\mathrm{CoA}$ 을 생성하여 자신의 위치를 홈 에이전트와 상대 노드에 게 등록한다. 즉 MIPv6에서, 이동 노드는 이동 노드가 움 직일 때마다 자신의 위치를 등록하는 과정을 수행한다. 이에 반하여 $\mathrm{WiBro}$ 는 새로운 $\mathrm{ACR}$ 로 이동시 자신의 위 치를 홈 에이전트나 상대노드에게 알리지만, $\mathrm{ACR}$ 내에서 의 이동일 경우에는 $\mathrm{ACR}$ 에게만 알리고 홈 에이전트나
상대노드에게는 알리지 않는다. $\mathrm{C}_{\mathrm{Mg}}, \mathrm{C}_{\mathrm{Wg}}, \mathrm{C}_{\mathrm{Ml}}, \mathrm{C}_{\mathrm{Wl}}$ 은 각 각 MIPv6와 WiBro에서 global binding update와 local binding update 시그널링 코스트를 나타낸다. IP 네트워크 에서, 시그널링 코스트는 두 네트워크 항목의 거리에 비 례한다. $\mathrm{C}_{\mathrm{Mg}}, \mathrm{C}_{\mathrm{Wg}}, \mathrm{C}_{\mathrm{Ml}}, \mathrm{C}_{\mathrm{Wl}}$ 은 아래 식으로부터 획득 된 다.

$$
\begin{aligned}
C_{M g} & =2 \cdot\left(k \cdot D_{A R-M N}+\tau \cdot D_{H A-A R}\right) \\
& +2 \cdot N_{C N} \cdot\left(k \cdot D_{A R-M N}+\tau \cdot\left(D_{H A-A R}+D_{C N-A R}\right)\right) \\
& +P C_{H A}+N_{C N} \cdot P C_{C N}
\end{aligned}
$$

$$
\begin{aligned}
C_{W g} & =2 \cdot\left(k \cdot D_{R A S-M N}+\tau \cdot\left(D_{H A-A C R}+D_{A C R-R A S}\right)\right) \\
& +2 \cdot N_{C N} \cdot\left(k \cdot D_{R A S-M N}+\tau \cdot\left(D_{H A-A C R}+D_{C N-A C R}\right)\right) \\
& +P C_{H A}+N_{C V} \cdot P C_{C N}+P C_{A C R}
\end{aligned}
$$

$$
C_{W}=2 \cdot\left(k \cdot D_{R A S-M N}+\tau \cdot D_{A C R-R A S}\right)+P C_{A C R}
$$

어디든 $\tau$ 와 $\mathrm{k}$ 은 각각 유선과 무선 링크에서 전송 코 스트 단위이다. $\mathrm{PC}_{\mathrm{HA}}, \mathrm{PC}_{\mathrm{CN}}$, 그리고 $\mathrm{PC}_{\mathrm{ACR}}$, 은 $\mathrm{HA}, \mathrm{CN}$ 그 리고 $\mathrm{ACR}$ 에서 binding update 처리를 위한 처리 코스트 이다. b,c,e 그리고 f은 노드 사이의 홉 거리를 나타낸다. $\mathrm{N}_{\mathrm{CN}}$, 은 $\mathrm{MN}$ 와 통신하는 $\mathrm{CN}$ 의 수 을 나타낸다.

WiBro은 ACR(Access Control Router)라고 불리는 local 에이전트를 사용하는 시그널링 코스트를 최소화하 여 성능을 향상시킨 것이다. $\mathrm{ACR}$ 은 라우터의 계층 구조 의 어느 레벨이든지 위치되어 질 수 있다. WiBro에서 $\mathrm{ACR}$ 은 도메인내 에서 하위 네트워크 주변으로 움직였을 때, $\mathrm{MN}$ 은 단지 현재의 $\mathrm{ACR}$ 에게만 $\mathrm{BU}$ 메시지를 보낸다.

랜덤 walk mobility model의 항목에서, MN가 global binding update을 수행하는 확률은 다음을 따른다 :

$$
P_{D, D} \cdot \alpha_{d, d+1}
$$

특히, 만약 MN가 D ring 범위로 구성되어진 $\mathrm{ACR}$ 도 메인의 경계링 과 $\mathrm{D}$ ring 범위에 위치한다면, ring $\mathrm{D}$ 의 범 위로부터 ring $\mathrm{D}+1$ 의 범위로 움직임을 수행했다. 그때 $\mathrm{MN}$ 은 global binding update 처리를 수행한다. 다른경우 에, 이 움직임을 제외하면, $\mathrm{MN}$ 은 단지 local binding update 처리를 수행한다. 그러므로, 일반적인 경우의 location update cost 와 시간 단위마다 제안되어진 방법은 다음의 식으로 표현되어 진다.

$$
C_{M L}=\frac{P_{D, D} \cdot \alpha_{D, D+1} \cdot C_{M g}}{T}
$$




$$
C_{W L}=\frac{P_{D, D} \cdot \alpha_{D, D+1} \cdot C_{W g}+\left(1-P_{D, D+1} \cdot \alpha_{D, D+1}\right) \cdot C_{W}}{T}
$$

어디든 $\mathrm{T}$ 은 평균 셀 거주 시간을 나타낸다.

\subsection{2 packet delivery cost}

$\mathrm{MIPv}$ 6와 WiBro에서 패킷 전달 비용 $\mathrm{C}_{\mathrm{MP}}, \mathrm{C}_{\mathrm{WWP}}$ 은 다 음의 식으로 계산 된다.

$$
\begin{aligned}
& C_{M P}=C_{H A}+C_{C N-M N} \\
& C_{W W P}=C_{A C R}+C_{H A}+C_{C N-M N}
\end{aligned}
$$

식 (22)에서, $\mathrm{C}_{\mathrm{HA}}$ 은 $\mathrm{HA}$ 에서 패킷 처리를 위한 처리 비 용을 나타낸다. $\mathrm{C}_{\mathrm{CN}-\mathrm{MN}}$ 은 $\mathrm{CN}$ 으로부터 $\mathrm{MN}$ 까지의 패킷 전송 비용을 나타낸다. $\mathrm{WiBro}$ 에서, $\mathrm{ACR}$ 은 매핑 테이블 을 관리하고, $\mathrm{MN}$ 의 현재 위치(LCoA)을 트랙하기 위하여 사용되어졌다. $\mathrm{MN}$ 를 가리키는 모든 패킷은 $\mathrm{HA}$ 에 의해 서 받아질것이고, 매핑 테이블을 사용하여 $\mathrm{MN}$ 의 $\mathrm{CoA}$ 로 터널 되어진다. 그러므로 lookup time은 매핑 테이블을 위하여 요구되어지고 고려되어지는 것이 필요하다. 특히, 패킷이 ACR에 도착되었을 때, $\mathrm{ACR}$ 은 매핑 테이블로부 터 목적지 $\mathrm{MN}$ 의 현재 $\mathrm{CoA}$ 을 선택하고, 패킷은 $\mathrm{MN}$ 로 라우트 되어진다. 그러므로 $\mathrm{ACR}$ 에서 처리 비용은 lookup cost $\left(C_{\text {lookup }}\right)$, 라우팅 비용 $\left(C_{\text {routing }}\right)$ 으로 나누어진 다. lookup cost는 매핑 테이블의 크기에 비례되어진다. 매핑 테이블의 크기는 도메인의 범위내에 위치되어진 $\mathrm{MN}$ 의 수에 비례한다[11]. 다른 한편으로, 라우팅 비용은 특별한 도메인에 속하는 $\mathrm{AR}$ 의 수의 대수에 비례한다 [12]. 식(24)에서, S은 패킷의 단위에서 평균 세션 사이즈 를 $\lambda_{s}$ 는 세션 도착율을 나타낸다. $a$ 와 $\beta$ 는 weighting 요 소이다. $\mathrm{N}_{\mathrm{MN}}$ 은 도메인에 위치되어진 유저의 총 수이다. 이 논문에서 우리는 $\mathrm{AR}$ 의 범위에 위치된 유저의 평균수 는 K 라고 가정하였다. 그러므로, 유저의 토탈 수는 식 (23)을 사용하여 획득되어질 수 있다.

$$
\begin{aligned}
N_{M N} & =N_{A R} \quad \times k \\
C_{A C R} & =\lambda_{s} \cdot S \cdot\left(C_{\text {lookup }}+C_{\text {routing }}\right) \\
& =\lambda_{s} \cdot S \cdot\left(\alpha N_{M N}+\beta \log \left(N_{A R}\right)\right)
\end{aligned}
$$

WiBro에서 route optimization은 삼각 라우팅 문제를 해결하기위하여 사용되어졌다. 그러므로 단지 세션의 첫 번째 패킷은 $\mathrm{MN}$ 가 다른 네트워크로 움직이는지 아닌지 를 감지하기 위여 $\mathrm{HA}$ 로 전송한다. 그 후에, 세션의 모든
연속하는 패킷은 직접적으로 $\mathrm{MN}$ 로 라우트 된다. $\mathrm{HA}$ 에 서 처리 비용은 다음과 같이 계산되어질수 있다 :

$$
C_{H A}=\lambda_{S} \cdot \theta_{H A}
$$

$\Theta_{\mathrm{H}}$ 은 HA에서 단위 패킷 처리 비용을 나타낸다. WiBro가 route optimization을 지원한 이후, WiBro에서 전송 비용은 식(26)을 사용하여 획득되어질 수 있다.

$$
\begin{aligned}
C_{C V-M N} & =\tau \cdot \lambda \cdot\left((S-1) \cdot\left(D_{C N-A C R}+D_{A C R-R A S}\right)\right. \\
& \left.+\left(D_{H A-C N}+D_{C N-A C R}+D_{A C R-R A S}\right)\right) \\
& +K \cdot \lambda \cdot S
\end{aligned}
$$

\section{4. 분석결과}

[표 1] 시스템 파라미터

\begin{tabular}{|c|c|c|c|c|c|c|}
\hline $\begin{array}{c}\text { Parameter } \\
\text { value }\end{array}$ & $\alpha$ & $\beta$ & $\tau$ & $\mathrm{k}$ & DHA-ACR & DCN-ACR \\
\hline Parameter & $\begin{array}{c}\text { DHA-C } \\
\mathrm{N}\end{array}$ & $\mathrm{N}_{\mathrm{CN}}$ & $\mathrm{PC}_{\mathrm{HA}}$ & $\mathrm{PC}_{\mathrm{CN}}$ & PCACR & DACR 1-ACR2 \\
value & 6 & 2 & 24 & 6 & 10 & 1 \\
\hline Parameter & $\begin{array}{c}\text { DRAS- } \\
\text { MN }\end{array}$ & $\begin{array}{c}\text { DACR- } \\
\text { RAS }\end{array}$ & $\lambda 1$ & $\lambda 2$ & & \\
value & 1 & 2 & 0.1 & 0.2 & & \\
\hline
\end{tabular}

표 1은 성능분석을 위한 파라미터 값이다[6,7,11].

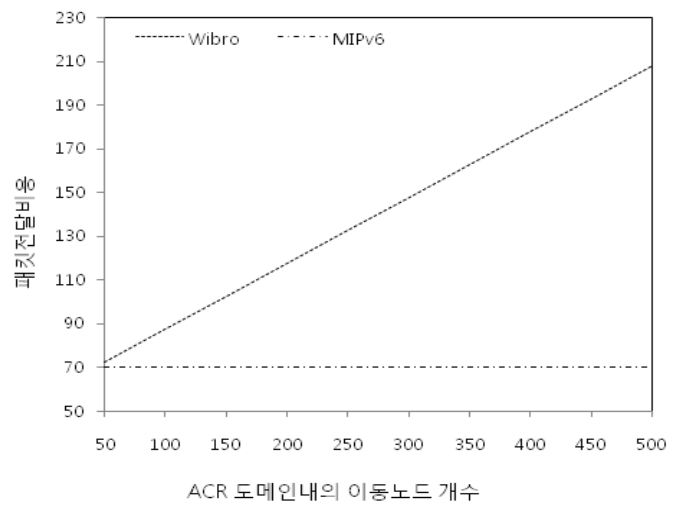

[그림 5] ACR 도메인 내의 이동 노드 개수에 따른 이동 노드 당 패킷전달비용

그림 5 는 도메인 $\mathrm{ACR}$ 안에서 이동노드의 증가에 따른 각 이동 노드당 패킷 전달 비용을 나타낸 것이다. WiBro 
의 도메인 $\mathrm{ACR}$ 안에 이동 노드가 증가할수록 패킷 처리 비용은 일정하게 증가한다, 반면, MIPv6의 경우 패킷 처 리비용은 일정하다. 이는 $\mathrm{WiBro}$ 의 $\mathrm{ACR}$ 의 패킷 처리비용 은 식(24)에서와 같이 이동노드의 개수에 비례하기 때문 에 이동노드가 증가하면 패킷 처리비용도 일정하게 증가 하는 것이다.

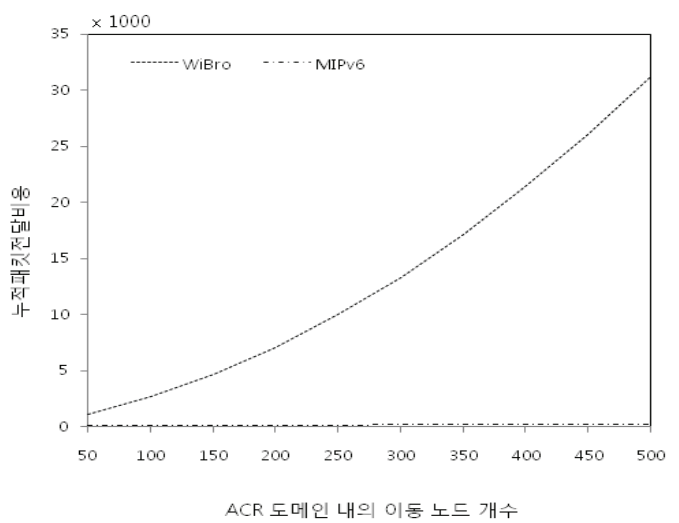

[그림 6] ACR 도메인 내의 이동 노드 개수에 따른 누적 패킷전달비용

그림 6은 $\mathrm{WiBro}$ 의 $\mathrm{ACR}$ 도메인 내에서 이동 노드의 개수에 따라 모든 이동 노드의 패킷 처리비용을 합한 것 이다. WiBro의 ACR 도메인 내에서 누적 패킷 처리비용 이 이동 노드 수의 제곱에 비례하고, MIPv6은 이동 노드 의 수에 비례한다.

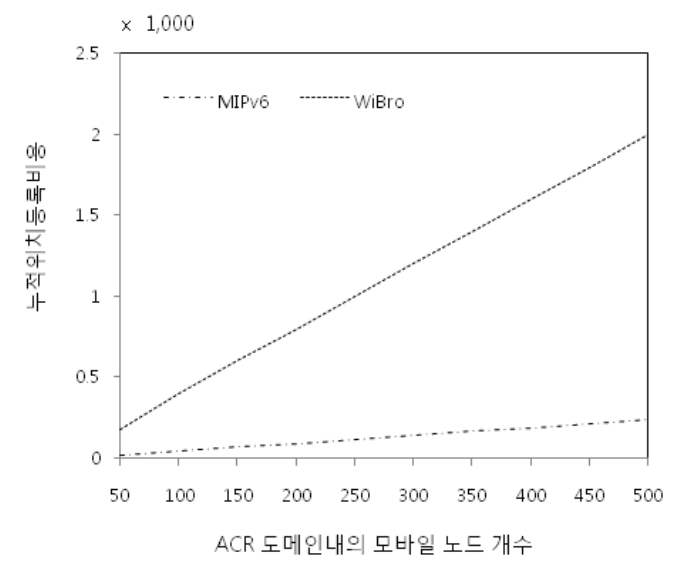

[그림 7] ACR 도메인 내의 이동 노드 개수에 따른 누적 위치등록비용

그림 7은 각 이동 노드의 위치 등록비용을 합한 것이
다. WiBro 와 MIPv6의 누적 위치 등록비용이 일정하게 증가한다.

\section{5. 결론}

정지 또는 이동 중에도 고속인터넷 접속이 가능한 무 선인터넷 규격인 $\mathrm{WiBro}$ 는 유선망이 갖는 기존 시스템의 한계를 극복하여 최소 시속 $60 \mathrm{Km}$ 이하의 이동성을 지원 하며 실내외에서 끊김없이 초고속 무선인터넷 서비스를 제공할 수 있도록 설계되어있다. 그러나 PSS와 RAS을 제어하고 IP패킷을 라우팅하는 $\mathrm{ACR}$ 은 이동노드로 송수 신되는 모든 패킷을 전달하므로, 이동노드의 개수가 증가 하면 패킷처리비용이 증가한다. 본 논문에서는 WiBro의 $\mathrm{ACR}$ 성능을 마코프 체인을 이용하여 분석하였다. 분석 결과, $\mathrm{ACR}$ 안에 이동 노드가 증가 할수록 $\mathrm{MIPv6}$ 의 경우 는 패킷 처리비용이 일정하지만, WiBro는 이동노드의 증 가에 따라 일정하게 증가한다. 따라서 누적 패킷처리비용 이 MIPv6에서는 이동노드의 수에 비례하여 증가하지만 $\mathrm{WiBro}$ 는 이동노드수의 제곱에 비례함을 볼 수 있으며 위 치등록비용은 $\mathrm{WiBro}$ 와 $\mathrm{MIPv} 6$ 가 각각 일정하게 증가함 을 볼 수 있다.

\section{참고문헌}

[1] D. Johnson, C. Perkins and J. Arkko,"Mobility Support in IPv6," RFC 3775, Jun. 2004.

[2] H. Soliman, C. Castelluccia, K. E. Malki and L. Bellier, "Hierarchical MIPv6 (HMIPv6) mobility management," Internet Draft, Nov. 2001.

[3] R. Koodi “ Fast Handovers for Mobile IPv6," RFC 4068, Jul. 2005.

[4] 송석일 외 2인, “초고속 WiBo 기술”, ETRI 전자통신동 향분석

[5] 인지환, 양정록, 김영일, “초고속 WiBro 기술”, 대한전 자공학회지, 2004.

[6] M. Woo, "Performance analysis of mobile IP regional registration, " IEICE Trans. Commun., vol. E86-B, no.2, pp.472-478, Feb. 2003.

[7] X. Zhang, J. G. Castellanos, and A. T. Capbell, "P-MIP: Paging extensions for mobile IP", ACM Mobile Networks and Applications, vol.7, no.2, pp.127-141, 2002.

[8] J. S. M. Ho and I. F. Akyildiz, "Mobile user location update and paging under delay constraints, 
"ACM-Baltzer J.Wireless Networks, Vol.1, pp413-425, 1995.

[9] Y.-B. Lin, "Reducing location update cost in a PCS network," IEEE/ACM Trans Networking, vol.5, pp.25-33, 1997.

[10] I. F. Akyildiz and W. Wand,"A dynamic location management scheme for next-generation multitier PCS systems," IEEE Trans. Wireless Commun., vol.1, no.1, pp. 178-189, Jan. 2002.

[11] Sangheon Pack and Yanghee Choi, "A Study on performance of hierarchical mobile IPv6 in IP-based cellular networks," IEICE Trans. Commun., vol. E87-B no.3 pp.462-469, Mar. 2004.

[12] J. Xie and F. Akyildiz, "A novel distributed dynamic location management scheme for minimizing signaling costs in Mobile IP, "IEEE Trans. Mobile Computing, Vol. 1, No, 3, pp. 163-175, Sep. 2002.

박 원 길(Won-Gil Park)

[정회원]

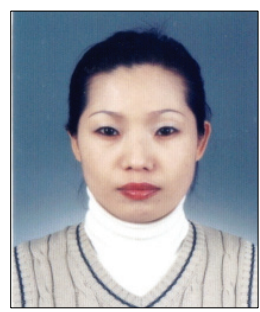

- 1992년 2월 : 호원대학교 전자계 산학과 이학사

- 1996년 8월 : 전북대학교 전자계 산교육학과 교육학석사

- 2007년 8월 : 숭실대학교 컴퓨터 학과 공학박사

- 2008년 3월 2009년 2월 : 한 국건설기술연구원 박사후 연구원

- 2009년 3월 현재 : 성균관대학교 유비쿼터스 컴퓨팅 기술연구소 선임연구원

<관심분야>

멀티미디어 통신, 이동 통신, 무선 센서 네트워크

\section{김 형 진(Hyoung-Jin Kim)}

[정회원]

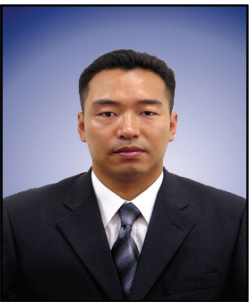

- 1997년 2월 : 호원대학교 전자계 산학과 이학사

- 1999년 8월 : 군산대학교 정보통 신공학과 공학석사

- 2004년 8월 : 군산대학교 정보통 신공학과 공학박사

- 2004년 9월 2005년 3월 : 군 산대학교 전자정보공학부 계약 교수

- 2005년 4월 2008년 2월 : 익산대학 정보통신과 조 교수

- 2008년 3월 현재 : 전북대학교 응용시스템공학부 조 교수

<관심분야>

멀티미디어 통신 시스템, 무선 센서 네트워크, 이동 통신 